\title{
STRUCTURAL CUES FOR SYMMETRY, ASYMMETRY AND NON-SYMMETRY IN CENTRAL TAURUS SIGN LANGUAGE
}

\author{
RABIA ERGIN $^{*}$, ANN SENGHAS ${ }^{2}$, RAY JACKENDOFF $^{3}$, and LILA GLEITMAN ${ }^{4}$ \\ ${ }^{*}$ Corresponding Author: Rabia.Ergin@mpi.nl \\ ${ }^{1}$ Max Planck Institute for Psycholinguistics, Nijmegen, The Netherlands \\ ${ }^{2}$ Department of Psychology, Barnard College, New York City, USA \\ ${ }^{3}$ Department of Brain and Cognitive Sciences, MIT, Cambridge, USA \\ ${ }^{4}$ Department of Psychology, University of Pennsylvania, Philadelphia, USA
}

Central Taurus Sign Language (CTSL) is a village sign language used in three villages in an isolated area in south-central Turkey. This language emerged in the absence of a linguistic model within the last half-century as a result of recessive deafness in these communities, which have deaf populations of $4.8 \%$, $.6 \%$ and $.5 \%$. CTSL provides us with a novel vantage point into how a brandnew system emerges and develops because it is relatively young, still evolving, and the very first creators of this system are still alive today.

Previous research has shown that symmetrical and reciprocal predicates have specific and distinct syntactic properties in spoken languages (Gleitman, Gleitman, Miller \& Ostrin, 1996) and in Nicaraguan Sign Language (NSL) (Flaherty, Goldin-Meadow, Senghas, Coppola \& Gleitman, 2013; Flaherty, Goldin-Meadow, Senghas, Coppola \& Gleitman, 2014). The present study investigated (i) whether CTSL has any distinctive structural cues marking symmetrical actions (e.g., shaking hands) and reciprocal actions (e.g., pushing each other); (ii) if it has, how these structural markers evolve over time across age cohorts of CTSL signers. We developed a controlled elicitation task in which deaf signers viewed 62 short clips and described them to a deaf/hearing addressee, who then picked the corresponding picture from an array of three pictures for comprehension check. The clips involved two characters performing actions that were symmetrical (e.g., shaking hands), reciprocal (e.g., punching each other), transitive (e.g., one punching the other), and intransitive (e.g., both punching towards the camera). Here we use symmetrical for events that are necessarily symmetric, reciprocal for events that are symmetric but can also appear in asymmetric/non-symmetric contexts, transitive for non-symmetric two- or three-argument events, and intransitive for non-symmetric one-argument events. Twelve signers, grouped into three successive age cohorts, participated $\left(M_{\text {age }}=42.2\right.$, age range: $\left.17-55\right)$. We detected several devices that were candidate 
structural cues, and compared them across cohorts as a measure of how CTSL has evolved over its 50-year existence.

Our findings, based on a total of 946 responses $\left(\mathrm{n}_{\text {intransitive }}=255, \mathrm{n}_{\text {_transitive }}=\right.$ $364, \mathrm{n}_{\text {reciprocal }}=190, \mathrm{n}_{\text {symmetrical }}=137$ ), are as follows: (1) Body segmentation: Symmetrical and reciprocal actions come to be frequently marked by body segmentation -one side of the body is allocated for one of the characters and the other side is allocated for the other character- but not transitive actions (Figure 1). (2) Mirroring: Signing with both hands in a mirror-image configuration (cf. Flaherty et al, 2014) was often used for reciprocal and symmetrical actions, but not for intransitive and transitive actions (Figure 2). (3) Temporal sequencing: CTSL signers distribute information temporally across an utterance by sequentially signing each action performed by each character in the contexts involving intransitive, transitive and reciprocal actions, but not symmetrical actions. Temporal sequencing becomes more systematic in successive cohorts from CTSL-1 to CTSL-2 $\left(\chi^{2}(1)=43.4, p<0.0001\right)$ (Figure 3). (4) Perspective: Flaherty et al. (2013) found evidence for double perspective verb pairs in reversible transitive contexts in NSL -events being expressed both from the agent's and the patient's perspective. In contrast, here we find that CTSL prefers a single perspective in reversible transitive events, but a double perspective in reciprocal and symmetrical events, with an increasing tendency for systematicity in reciprocal events from CTSL-1 to CTSL-2 $\left(\chi^{2}(1)=48.4, p<0.0001\right)$ (Figure $4)$.

Briefly, our results indicate that, first, body segmentation and mirroring are strong tendencies as of CTSL-1 because signers makes use of the iconicity of the body, whereas temporal sequencing and perspective take time to invent as structural markers. Second, reciprocal and symmetrical actions differ from transitive actions in that the transitive actions do not use body segmentation and mirrored articulators, and, they are expressed from a single perspective, whereas reciprocal and symmetrical actions are body segmented, mirrored, and expressed from double perspectives. Third, reciprocal and symmetrical actions differ from plural intransitives by mirror-image configuration. Fourth, a reciprocal action differs from a symmetrical action in that the former is temporally sequenced whereas the latter is not. All in all, each action has its own combination of essential components to encode symmetry, asymmetry, and non-symmetry, and these components become more conventionalized across cohorts. Our findings provide further evidence for how a brand-new language converges on distinctive shared devices that differentiate between verb classes in an increasingly systematic way over generations of learners. More broadly speaking, the rapid cultural development of linguistic expressions that distinguish reciprocal and symmetrical actions from transitive actions and from each other suggests that a sensitivity to the semantics of these distinctions is present in the language-ready brain, and that this sensitivity is a product of biological evolution of the human linguistic and/or conceptual capacity. 


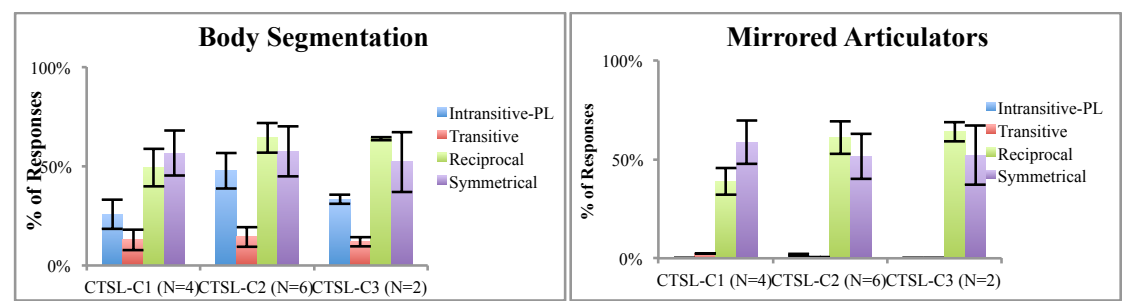

Figure 1. Overall proportion of body. seg. across cohorts
Figure 2. Overall proportion of mirrored articulators across cohorts

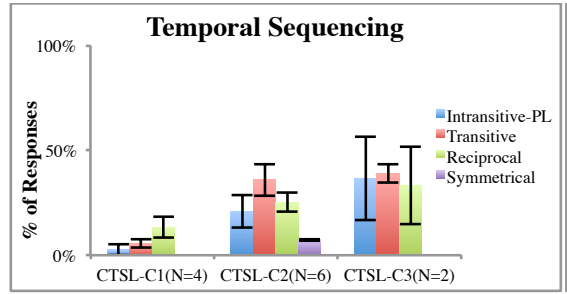

Figure 3. Overall proportion of temp. seq. across cohorts

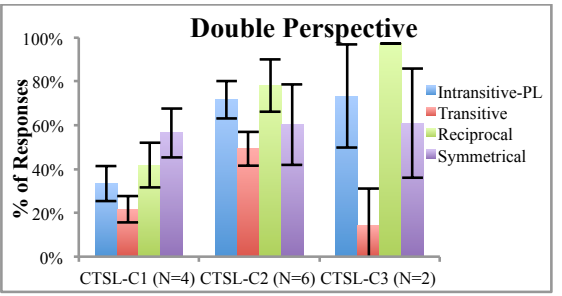

Figure 4. Overall proportion of double. perspective across cohorts

\section{References}

Flaherty, M., Goldin-Meadow, S., Senghas, A., Coppola, M. \& Gleitman, L. (2014, April). Language from Gesture? Emergent Transitivity Marking in Nicaraguan Sign Language. Paper presented at the $10^{\text {th }}$ Evolution of Language Conference, Vienna, Austria.

Flaherty, M., Goldin-Meadow, S., Senghas, A., Coppola, M. \& Gleitman, L (2013, November). Animacy and Verb Classes in Nicaraguan Sign Language. Poster presented at the $38^{\text {th }}$ Annual Boston University Conference on Language Development, Boston, MA.

Gleitman, L., Gleitman, H., Miller, C., \& Ostrin, R. (1996). 'Similar' and similar concepts. Cognition, 58, 321-376. 